\title{
DISCUSSION OF OBSERVATIONS OF THE FLOW OF \\ MATTER WITHIN BINARY SYSTEMS
}

\author{
ALAN H. BATTEN \\ Dominion Astrophysical Observatory, Victoria B. C.
}

\begin{abstract}
Observational determinations of density, dimensions, temperatures, and velocities of circumstellar features are surveyed and discussed, with a view to establishing limiting values that could be useful in any theoretical treatment of circumstellar structure. Densities of the order of $10^{13}$ particles $\mathrm{cm}^{-3}$ are found for streams and disks, although there is evidence for regions of much lower density in many systems, and some systems may have much denser circumstellar matter.

Dimensions of disks seem to be remarkably constant (fractional radius $\sim 0.3$ ) in a wide variety of systems. The total mass of circumstellar matter is always a small fraction of the mass of the system. Temperatures are usually similar to the temperatures of the stars in the system, although hot spots certainly exist in some systems, and 'flare' activity is evidence of localized very high temperatures. On the other hand, some systems may contain solid circumstellar matter. Observed velocities are usually several hundred $\mathrm{km} \mathrm{s}^{-1}$. The empirical relation between the velocity of rotation of disks and the orbital period is described. Finally, the stability of these features is briefly discussed.
\end{abstract}

\section{Introduction}

The observational evidence for the existence of circumstellar matter in close binary systems was reviewed recently (Batten, 1970) and this review has been updated, amended, and extended in a book to be published shortly (Batten, 1973). Despite great activity in the field within the past eighteen months, which has made this Symposium seem even more timely than it appeared to be when it was first proposed, a second updating now of my 1970 review would contain more repetition than could be reasonably justified. Therefore, in this review, I shall try to examine both new and old papers in order to find out what is known about the physical properties of circumstellar material in binary systems and to determine what constraints the presently available observations place on any theory of the structure and motions of this circumstellar matter.

In the 1970 paper, I introduced the concept of three elements in the circumstellar matter: disk, streams, and cloud (see Figure 1 of that paper). This concept still seems to me to be useful, and I shall use the same terminology in this review. There are not of course, sharp density boundaries between the different elements, as is inevitably suggested by the diagram: the real distinctions between elements are probably kinematic, and even these cannot be sharp. Nevertheless, densities within a region several stellar radii above the surface of one component of a binary system are likely to be very much less than those within a stream flowing between the components. Even within a stream, there may be great variations in density, depending on the velocity of the material within the stream (Korsch and Walter, 1969). The distinction between these three elements is certainly important in discussions of circumstellar density, however. Unfortunately, not every investigator has made it clear to which part of a binary system his estimate of circumstellar density applies. One recent review included erroneous conclusions about the capacity of the observed streams for mass transfer 
partly because a 'mean stream density' was derived from figures referring to all three elements. From the title of this paper, which is based on that laid down by the Organizing Committee, you may expect me to concentrate on a discussion of the streams, but it seems to me to be necessary to consider all three elements of the circumstellar matter.

New observational tools are now at our disposal that will undoubtedly prove powerful aids in the study of circumstellar matter, and greatly contribute to our understanding of it. The first observations of a close binary system ( $\beta$ Lyrae) made from above the Earth's atmosphere have been published (Kondo, 1971; Kondo et al., 1972) and many more have been obtained by the OAO. Observations of the far ultraviolet region of the spectrum have proved of great help in understanding the loss of mass from stars of early spectral type (Morton 1967a, b; Morton et al., 1968; Hutchings, 1970) and they may also be expected to contribute decisively to our knowledge of mass exchange within binary systems. Unfortunately, many of the most interesting systems are rather faint for the instruments presently above the atmosphere of the Earth. There is also increasing evidence for the identification of several galactic X-ray sources with close binary systems (Burbidge et al., 1967; Kristian et al., 1967; Kraft and Demoulin, 1967; Murdin and Webster, 1971, 1972; Bolton, 1972a; and - less certainly - Braes and Miley, 1972; van den Bergh, 1972). Prendergast and Burbidge (1968) have shown that X-rays could be generated by streaming circumstellar matter - a hypothesis that has been further elaborated by Kraft (1972). Finally perhaps the greatest innovation has been the detection of radio waves from four close binary systems: Antares B, $\beta$ Lyrae, $\beta$ Persei, and HD 226686 (probably Cyg X-1). The clearest observational picture presently available is that for $\beta$ Persei (Wade and Hjellming, 1972; Hughes and Woodsworth, 1972). This indicates that a kind of flaring activity takes place at times within the system, and this may be paralleled by activity in the optical spectrum (Bolton, 1972b). These are exciting developments indeed, but it is still difficult to integrate them into our already existing corpus of knowledge. There is no doubt that these new results will figure prominently in our discussions, but I shall base my review primarily on the results of ground-based, conventional observations which have so far contributed the greatest part of our knowledge of circumstellar matter in binary systems. The last word, however, at least for some systems, may come from these new observations in spectral regions very different from those employed in conventional spectroscopy and photometry, or from observations in the normal spectral regions made with very much higher time resolution than is now possible.

\section{Location and Dimensions of the Elements of Circumstellar Matter}

Disks are usually detected by their emission spectra which, in many systems, are best seen at primary eclipse (when the star within the disk is hidden). The behavior of these emission lines during eclipse clearly reveals that they arise in some structure that surrounds the eclipsed (usually primary, or more massive) star. The classic example is RW Tauri, but disks are known in systems of widely different sorts, from short- 
period systems containing high-temperature stars, through the Algol-type systems (including RW Tauri) to systems like SX Cassiopeiae and VV Cephei that contain giant or supergiant stars. For the Algol-type systems, crude estimates of the radii of the disks can be made from the duration of the visibility of the emission lines during eclipse. Many of these have been quoted elsewhere (Batten, 1970). Plavec (1968) developed a more refined method for determining the size of the disk from the width of the line profile. This method is based on the assumption that individual particles within the ring rotate in Keplerian orbits. He found that the disk in RW Tauri must have a radius of about 2.6 times the radius of the star that it surrounds. Baldwin (1972) applied the same analysis to observations of U Cephei, and found that the disk had a radius of 2.8 stellar radii (or about 8.4 solar radii).

In the much larger system of SX Cassiopeiae, Günther (1959) has estimated that the disk has a radius of about 4.6 times that of the primary star. He did this by comparing the durations of primary and secondary eclipse, assuming that the disk is opaque enough to eclipse the secondary star, but that its own eclipse (in the light of the continuous spectrum) is not perceptible. Another long-period system, AR Pavonis, has been discussed by Thackeray (1959). From the variation of the emission-line profile during eclipse, he finds that the primary star must be surrounded by an extended disk, about twice the radius of the secondary star, that is both expanding and rotating.*

The spectrum of VV Cephei shows very strong emission lines of hydrogen which are again interpreted as arising in a structure surrounding the (invisible) hotter component, a star probably of late $\mathrm{O}$ or B spectral type. Model computations by Hutchings and Wright (1971) suggest that this the denser part of the structure has a radius of about 50 times the radius of the hot star, which they believe to have a radius of $13 R_{\odot}$, but the whole structure extends to about 150 times the radius of the hot star (the separation of the two stars appears to be about $5400 R_{\odot}$, and the radius of the M-type star to be about $1800 R_{\odot}$ ). This structure perhaps should not be called a disk, since they estimate that it fills about 85 per cent of the spherical region of radius $1950 R_{\odot}$ that surrounds the primary star.

Disks are also observed in the eruptive binaries where they may account for a significant fraction of the total light. These systems, for the most part, have very short periods and very small orbits. Disks in these systems seem to have radii approximately equal to the Sun's radius (Smak, 1971a; Kraft, 1959). The central stars in the disks are probably white dwarfs, at least in the systems DQ Herculis and U Geminorum. The separation of the two stars in the latter system, although very uncertain, is estimated to be about $3 R_{\odot}(\mathrm{Smak}, 1971 \mathrm{a})$.

If the radius of the disk is denoted by $\varrho$, and the major semi-axis by $a$, then we can use the quotient $\varrho / a$ as a measure of the size of the disk relative to that of the system. If $\mathrm{U}$ Geminorum is typical of eruptive binaries, then $\varrho / a$ is approximately 0.3 in these systems. In the Algol-type systems it appears to be somewhat larger, namely about 0.6 - again if U Cephei and RW Tauri can be considered typicat, but the method of

* See, however, his own comments on p. 98. 
determination used for these two systems will necessarily give a larger result than would be obtained by other methods. In VV Cephei, $\varrho / a$ is approximately 0.35 . Brown et al. (1970) have measured, with their stellar interferometer, the angular diameter of the emitting region that surrounds the Wolf-Rayet component of $\gamma^{2}$ Velorum. They find $\varrho / a=0.24$. Thus $\varrho / a$ seems to be nearly constant at about 0.3 in many different kinds of systems. Only in the Algol-type systems does the disk seem to be larger relative to the size of the system. Even in these systems, the disks are larger only by a factor of two that may well be accounted for by observational uncertainty.

Even less is known about the extent of these disks above or below the orbital plane. The convention, at least in the Algol-type systems, has been to assume that the circumstellar matter is highly concentrated toward the orbital plane. The famous representation of the 'ring' in RW Tauri (Skilling and Richardson, 1947) shows a very thin ring indeed - but this was only ever intended as a rough qualitative representation. Unfortunately, it has become fixed in many people's minds as a real indication of the nature of these rings or disks. Batten (unpublished) has found evidence for the existence of fairly substantial disk, in U Cephei, which scatters an appreciable amount of the light of the primary star and must therefore cover a considerable portion of its surface. These disks, therefore, must have a thickness (perpendicular to the orbital plane) that is at least comparable to the diameter of the central star. On the other hand, since the disks are rotating rapidly (except in the long-period systems, see Section 6) they are likely to be very appreciably flattened, and therefore to extend much less above and below the orbital plane than they do within it. Figure 1, although still schematic, probably gives a better idea of the sort

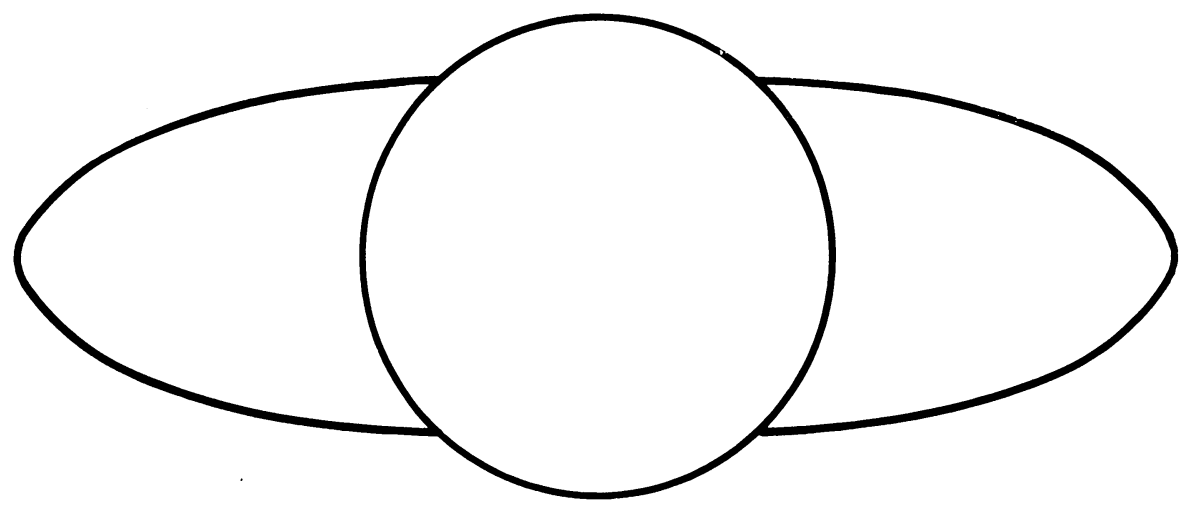

Fig. 1. Schematic representation of a disk in a typical Algol system.

of structure that seems to exist in many Algol-type systems. Disks of similar size, although possibly of somewhat different character (Section 5), have been proposed for a number of systems by Hall and his colleagues (e.g. Hall, i969; Hall and Taylor, 1971). In the eruptive binaries, according to Smak (1971a) the disks are 'quite extended in the $z$-coordinate', but little is known. In VV Cephei, as we have already seen, the 
model by Hutchings and Wright indicates that the 'disk' nearly fills a large spherical volume, and therefore must extend quite far out of the orbital plane. It would seem most natural to assume that the structure is an oblate spheroid of revolution. Its rotation is much less than that of disks in short-period systems, and therefore the structure is much less flattened. Coyne (1970) has found from the variable polarization of light in $\beta$ Lyrae that the disk in that system must be appreciably flattened. None of these estimates is directly based on physical measurements and the extension of circumstellar disks outside the orbital plane is but poorly known. It is, indeed, difficult to determine this observationally, and the uncertainty in this quantity must reflect on any attempt to determine the masses of circumstellar disks.

Even fewer determinations have been made of the precise course and dimensions of streams. Many schematic diagrams have been published for particular systems, but they are nearly all qualitative, even including those that resemble, in appearance, the precision and detail of an anatomical drawing! Our ideas about the courses of streams are based very largely on the results of particle-dynamics calculations in which pressure effects within the streams, collisions between individual ions, and any possible magnetic forces are ignored. The most comprehensive theoretical calculations of trajectories yet made are based on these assumptions of particle dynamics (Plavec et al., 1964; Plavec and Krŕž, 1965). Prendergast (1960), Sobouti (1970), and Biermann (1971) have studied the hydrodynamical behaviour of circumstellar gases in binaries, but they have computed velocity fields rather than trajectories. The observer who wishes to compare his results with theory can make the comparison only with particledynamics trajectories. Surprisingly the comparison is not bad, although Kopal (1971a) argues that particle dynamics is insufficient even for a useful first approximation. The matter can only be settled by determining the entire trajectories (course and velocity) of streams in several systems. This is very difficult and perhaps impossible to do, but until it has been done arguments about whether particle dynamics is useful at all will remain academic. No doubt both hydrodynamic and magnetic effects are present in many systems (Walter, 1971a, 1972) and may dominate in some, but in most systems there seems to be little or no conflict between the observations of streams - as far as they have been interpreted - and the predictions of particle dynamics. Observations of disks are in a different category. Some disks have been observed which ought not to exist if the assumptions of particle dynamics were rigorously valid. The point of this argument is not to try to defend a theory that must be wrong in its predictions of details, but to urge that it is a useful first approximation.

Circumstellar matter is to be found not only around one of the components of a binary system, but also between the two components. This is attested by observations of emission at $\mathrm{H} \alpha$, in the spectrum of Algol (Struve and Sahade, 1957) and possibly also in the spectrum of U Coronae Borealis (Struve et al., 1957). This is often attributed to a concentration of matter near the Lagrangian point, but the emission could equally well arise from the whole length of the stream between the two components. In the case of Algol, however, variations in the intensity of the emission do seem to indicate an accumulation of matter near the Lagrangian point (Andrews, 1967). 
Other observations of the stream in Algol have been made by Fletcher (1964) who has detected its effect on the $\mathrm{He}$ I line at $\lambda 4471$ in the spectrum of the system. The observations show an increase in the amount of absorption in this line about seven hours before the middle of primary eclipse (or about $2 \mathrm{~h}$ before the eclipse begins). The absorption reaches a maximum about three hours before mid-eclipse and thereafter declines steeply to a sharp minimum at mid-eclipse, rising again to a secondary maximum about four hours later. The sharp decrease, of course, is caused by the eclipse of the brighter star by the fainter. The other variations were explained by Fletcher as the result of the eclipse of a gaseous stream, also by the fainter star. The stream absorbs extra light in the wavelength $\lambda 4471$, and therefore modifies the limbdarkening coefficient in that wavelength so that it is different both from that in the neighbouring continuum and from the value it would have in a pure stellar line. The observations can be represented by a tongue of matter contiguous with the secondary component. The extent of the stream above and below the orbital plane is shown by Fletcher's analysis to be comparable to its width within the orbital plane. Here is an attempt to map the stream in Algol at least semi-quantitatively, but the results do raise some questions. First, we expect on theoretical grounds that the stream does not originate from a whole hemisphere of the secondary component, but from a small area around the Lagrangian point of the, presumably, distorted secondary star. Second, we may question whether circumstellar matter so close to the G-type component of the system would be hot enough to absorb the radiation of a helium line in a detectable quantity - the observation of additional absorption at $\lambda 4471$ suggests that the bulk of the matter producing the absorption is in fact fairly close to the B-type

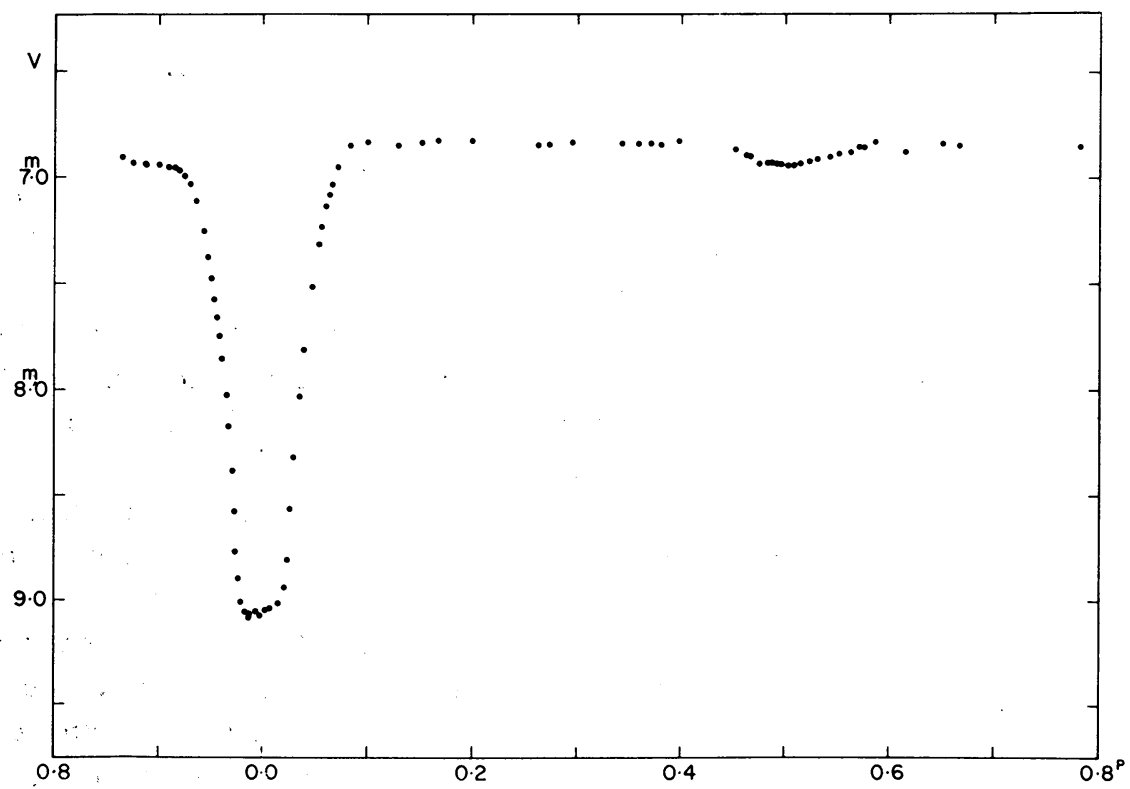

Fig. 2. The light curve of U Cep in $V$ light, as determined by Broglia (unpublished). 
component. Third, it is exceedingly implausible that a tongue of matter emerging from the G-type star should just stop. The matter must either fall into the B-type star, or go around it, and in either case it should still be detectable. These last two arguments suggest that the matter producing the extra absorption may be in a tongue extending from the B-type star, rather than from the G-type star, and it might be worthwhile to reconsider the same observations on the basis of a somewhat different model.

The light curve of $U$ Cephei provides a similar clue to the course of the stream in this system (Figure 2). Between phase 0.8 and 0.9 the total light of the system decreases by about $0 \mathrm{~m} .1$, and the shoulder preceding eclipse is lower than that following it by this amount (eclipse begins at 0.92 ). The disturbance of the light curve is the same in all colours, and can be traced right into eclipse, until phase 0.95 . The similarity of the disturbance in all colours suggests that it is caused by electron scattering in a stream that wholly or partially obscures the primary component at these phases, until it is itself eclipsed by the secondary star. The phases at which the disturbance appears and disappears define boundaries in the orbital plane between which the obscuring matter must lie (Figure 3). The extent of the stream perpendicular to the orbital plane cannot be derived from this information, but a plausible assumption is that the stream is roughly conical, the axis of the cone being aligned somewhere within the triangle of Figure 3. The observed diminution of $0^{m} \cdot 1$ in the light of the system would require

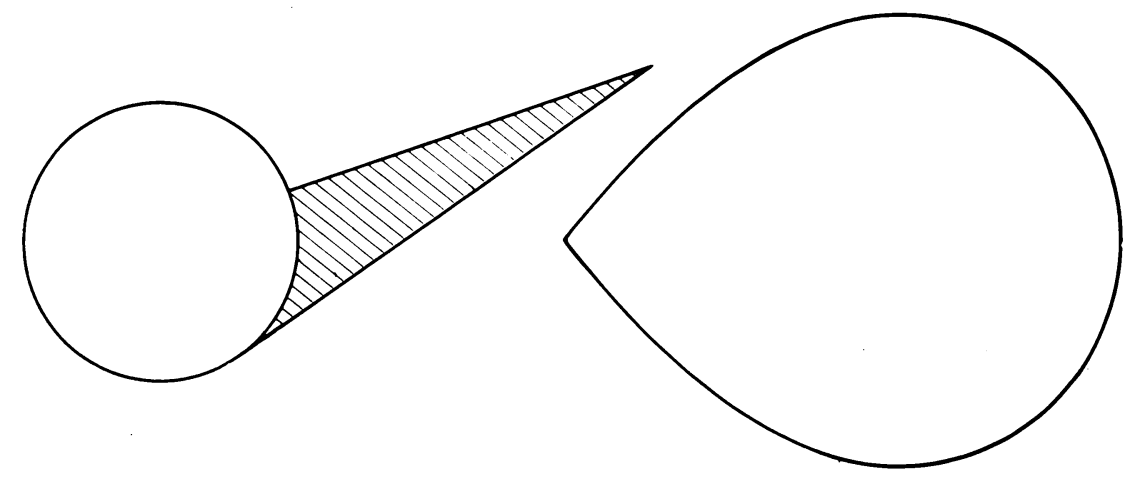

Fig. 3. Location of the stream in U Cep, as indicated by the light curve.

$1.4 \times 10^{23}$ scattering electrons above each square centimetre of the star's surface, if the absorption is taking place uniformly over the entire stellar disk. More probably only a portion of the stellar disk is covered. If we knew the length of the stream and its diameter, we could compute its density. The length cannot be much more than the distance between the surfaces of the two stars, say about twice the radius of the primary star, and a reasonable guess at the area of the stream where it meets the surface of the star is that it is approximately equal to the area of the projected disk of the Sun. The primary star has a radius of about three solar radii, so that these assumptions 
lead to a density for the stream of about $3 \times 10^{12}$ electrons $\mathrm{cm}^{-3}-$ somewhat lower than I have previously estimated (Batten, 1970, 1973) but still within the range found for stream densities. There is evidence that some of this stream goes right round the B-type star, and returns to the G-type star.

If the course deduced for the stream in U Cephei is correct, and if the matter does originate from the Lagrangian point of the secondary component, then somewhere it must make a sharp turn. Such a turn is compatible with particle-dynamics trajectories (Plavec et al., 1964) for particles that have been ejected tangentially to the surface of the secondary star, at fairly high velocities. It is not necessary to propose magnetic fields, although, of course, they may be present. Direct evidence of magnetic fields would be hard to obtain, because the spectrum of $U$ Cephei and those of most Algol-type systems, have such broad lines that Zeeman splitting would be almost impossible to detect. Indirect evidence might come from the careful determination of trajectories. Walter (1971b) has considered the trajectory of the stream in another Algol-type system, SW Cygni, and more recently (1972) in the system TW Andromedae. He finds that the light curve of this system cannot be interpreted without the assumption of additional light, and he supposes this light to arise in bright spots on the surface of the primary star, where the stream meets the surface. If this assumption is granted, then he finds that the bright spots are in quite high latitudes of the primary star (the equator being supposed to coincide with the orbital plane) and he suggests that the stream has been bent out of the orbital plane by magnetic fields. This hypothesis might not be necessary, however, if more were known about the density distribution within the stream, perpendicular to the orbital plane. Hall and Garrison (1972) have proposed a different model for this system. Other indirect evidence of the presence of magnetic fields may be provided by the radio observations of Algol (Hughes and Woodsworth, 1972). Appreciable local magnetic fields are very probably present within the streams, since they must be composed essentially of plasma. It is still not clear whether these local fields would have a large-scale effect on the trajectory of the stream.

Hansen and McNamara (1959) have investigated the stream trajectories in the system of RZ Scuti and have published a detailed, but qualitative, scheme of trajectories. They find evidence for a stream circulating the whole system, and for a large counter-rotating eddy in the neighbourhood of the following hemisphere of the primary star. This eddy is proposed to explain changes in the asymmetry of the heliumline profiles observed at phases 9 and 13 days (the orbital period is just over 15 days). These changes are in turn held responsible for step-like distortions of the velocity curve. Hansen and McNamara were the first to point out this step-like feature, although there are also hints of it in the velocity curve of U Cephei (Struve, 1960) and similar features are to be found in the velocity curve of VV Cephei (Hutchings and Wright, 1971). There is insufficient room in the system of $U$ Cephei for a counter eddy of the size proposed by Hansen and McNamara for RZ Scuti, and the velocity curve of VV Cephei would require several eddies for its explanation. These considerations throw some doubt on the explanation offered by Hansen and McNamara, and 
yet the steps do seem to be associated in some way with the presence of gas streams. These step-like features may be a useful clue to the study of the nature and structure of the streams.

Most of the little quantitative work that has been done on gas streams has been concerned with the Algol-type systems, but a model devised for UX Ursae Majoris a system that has much in common with the eruptive binaries - by Walker and Herbig (1954) should be mentioned. They found evidence for a stream completely detached from the hotter component, approximately half circling it (visible from phases $0^{P} .4$ to 0.8 ) from which matter is falling on to the hot star. Its width is comparable to the radius of that star, as is also the separation of the inner boundary of the stream from the surface of the star. The portion of the stream projected on the stellar surface between phases 0.7 and 0.8 is believed to be hotter than the rest.

The dimensions of clouds can often be roughly estimated from the dilution effects in their spectra. Clouds are the elements in circumstellar matter that display shell spectra. Although they are often drawn as detached shells, they are probably more nearly tenuous clouds that surround the system, or perhaps spirals of matter escaping the system, as Kuiper (1941) envisaged for $\beta$ Lyrae, or Herbig, Preston, Smak, and Paczyński (1965) have suggested for V Sagittae. They have traced out a spiral jet to some ten times the separation of the component stars, and Figure 15 of their paper indicates both the location and the width (in the orbital plane) that they have deduced for the jet from their measurements of the velocities and intensities of absorption reversals in the hydrogen emission lines of this complex spectrum. As the authors themselves point out, the reliability of the radial scale of the model depends strongly on the accuracy with the radial velocity can be determined from the absorption reversal. The maximum width of the jet within the orbital plane appears to be somewhat greater than the separation of the two stars.

Thackeray (1971) has studied the emission lines in the spectrum of HD $72754-\mathrm{a}$ system that seems to resemble $\beta$ Lyrae. The hydrogen and helium emission lines are cut into by absorption, and Thackeray finds that the combined profiles can be explained if they arise from a narrow, detached ring rotating around the whole system, and having a radius of four to five times the separation between the two stars. These features in the spectrum resemble the spectrum of $\mathrm{W}$ Crucis. Thackeray explicitly states that the ring is not expanding as the similar features in $\beta$ Lyrae and W Crucis seem to be.

In some systems that show shell spectra, the shell component seems not to arise from a shell, in any ordinary sense of that word, but from portions of streams that, because of the size of the system, are far enough from either of the stellar components to display a shell-type spectrum. This seems to be the case, for instance, in AX Monocerotis (orbital period 232.5 - see Plavec and Harmanec, 1972). Shell features appear in the spectrum only at certain orbital phases, when, presumably, the relevant part of the stream is exposed to view. A similar situation is encountered in the system of V 367 Cygni (Heiser, 1961) in which the shell component of the spectrum also seems to arise in a stream. The pattern of streaming in this system, however, is quite different 
from anything encountered in the Algol-type systems. The streams are centred on the primary component (of spectral type A2), apparently falling in on one side, and expanding outwards on the other.

\section{Density and Mass of Circumstellar Features}

The available evidence on the density of disks, streams, and clouds has already been summarized (Batten, 1970, 1973). Only a few new estimates have been published since the latter compilation. Walter (1971a) estimates an electron density of $10^{12} \mathrm{~cm}^{-3}$ for the stream in SW Cygni, and this fits well with my earlier conclusion that electron densities in the streams are of the order of $10^{13} \mathrm{~cm}^{-3}$, with an uncertainty of at least one in the exponent (which may be due either to mere uncertainty, or possibly to a real variation from system to system). Disks probably have similar but slightly lower densities, and clouds seem usually to have electron densities $10^{11} \mathrm{~cm}^{-3}$, or less.

Warner and Nather (1971), however, propose a much higher density for the streams in $\mathrm{U}$ Geminorum - namely, $1.5 \times 10^{16}$ electrons $\mathrm{cm}^{-3}$. This estimate is based on the observed period change, which is in turn interpreted as being caused by mass transfer between the components. They also estimate the density of the disk as $6 \times 10^{17}$ electrons $\mathrm{cm}^{-3}$. This is derived by assuming its mean lifetime, in the absence of any supply of matter, to be of the order of $10^{5} \mathrm{~s}$, as computed theoretically by Gorbatskij (1969) and by balancing this against the amount of matter supplied to the disk by the stream to obtain the mass of the disk. If a value is then assumed for the volume of the stream (they assume $10^{30} \mathrm{~cm}^{3}$ ) then the density can easily be derived. These densities derived by Warner and Nather are considerably higher than any other empirical estimates. While $U$ Geminorum is undoubtedly an unusual system, these high densities have not yet been found in any other eruptive binary. Such estimates as have been made are more in line with those made for 'ordinary' binaries, e.g. DQ Herculis $3 \times 10^{13}$ electrons $\mathrm{cm}^{-3}$ (Kraft, 1959), AE Aquarii $6 \times 10^{10}$ particles $\mathrm{cm}^{-3}$ (Crawford and Kraft, 1956), WZ Sagittae $4.5 \times 10^{12}$ electrons $\mathrm{cm}^{-3}$ (Krzeminski and Kraft, 1964). The estimate of stream density depends entirely on the period change being correctly identified as due to mass transfer. The rate of change is derived from observations extending over five years, or 10000 cycles. It is not clear whether the period may not be steadily increasing, or whether abrupt changes have occurred. Warner and Nather interpret the period change as a steady increase corresponding to a rate of mass transfer of $3 \times 10^{-7} M_{\odot} \mathrm{y}^{-1}$ or approximately $2 \times 10^{19} \mathrm{gm} \mathrm{s}^{-1}$ although even a steady change of period could be partly due to mass loss. Now Kraft (1972) in his discussion of $\mathrm{X}$-ray radiation from close binaries suggests that $\mathrm{X}$-rays are not observed from $U$ Geminorum stars because the rate of mass transfer is too low, and the rate he quoted is about $10^{16} \mathrm{gm} \mathrm{s}^{-1}$. Warner and Nather may have overestimated the mass transfer rate, and hence the density of the stream because the period change is still poorly determined (and perhaps misinterpreted). The high density of the disk depends on the density determined for the stream, and is therefore probably similarly overestimated. 
Smak $(1971 \mathrm{a}, \mathrm{b})$ has suggested that the observed period changes in eruptive binaries are not caused by mass transfer, but by interaction between the rotating disk and the star that it surrounds. Some eruptive binaries show irregular period changes, in either direction, of approximately $(\mathrm{d} \ln P / \mathrm{dt})=10^{-10}$ or $10^{-8} \mathrm{~d}^{-1}$. To produce changes of this magnitude in the way he has proposed, Smak finds that the disks must have at least $10^{-6}$, and possibly $10^{-4}$, of the mass of their central stars (generally assumed, in the eruptive binaries, to be white dwarfs of approximately solar mass). Even with the exceptionally high densities derived by Warner and Nather, and with the volume they assumed, the mass of the disk in U Geminorum comes to less than $10^{-9} M_{\odot}$. It therefore seems that the mechanism suggested by Smak cannot apply to eruptive binary systems.

The volumes of the disks in Algol-type systems are naturally larger - by a factor of the order of $10^{5}$. The estimated mean density of these disks, however, is of the order of $10^{13}$ electrons $\mathrm{cm}^{-3}$, and if ionization within the disk is complete, or nearly so, the mass of the disk again works out to be of the order of $10^{-9}$ or $10^{-8} M_{\odot}$. The mass of the disk in SX Cassiopeiae has recently been estimated at $10^{-9}$ to $10^{-6} M_{\odot}$ (Koch, 1972).

In the larger, long-period systems the disks are larger still. In VV Cephei the 'disk' is very large, but the density within it falls off very steeply (Hutchings and Wright, 1971). Its total volume is of the order of $10^{43} \mathrm{~cm}^{3}$. If it had a uniform density of $10^{11}$ particles $\mathrm{cm}^{-3}$, and if these particles were all hydrogen atoms, the total mass of the disk would be about $10^{30} \mathrm{gm}$ or $10^{-3} M_{\odot}$ (of the order of $10^{-4}$ of the mass of its central star). Because the density is increasing steeply inwards and is probably much higher in the central regions, the total mass of the disk must be greater than this. In this system, therefore, the disk is massive enough to interact gravitationally with the components of the binary. Its effect on period changes would be hard to detect, however, since the period is $20 \mathrm{y}$.

A very massive disk indeed was proposed for $\beta$ Lyrae (Woolf, 1965) comparable in mass to the central star itself. It is very doubtful whether so massive a disk would be stable. (In one sense no disks are stable: Gorbatskij's (1969) computations indicate disks would speedily collapse if no mass were supplied to them. A very massive disk, however, presents special problems of short-term stability.) Shulov (1967) attempted to derive the density and mass of the disk in $\beta$ Lyrae from his polarization measurements and found a total mass for the disk of $10^{-7} M_{\odot}$. In view of the strength of the emission lines in the spectrum of $\beta$ Lyrae, which are usually attributed to the disk, this may seem a rather low mass, but even if the disk is a scaled down version of that found in VV Cephei, it must be several orders of magnitude less massive than was suggested by Woolf.

Hall (1971) has proposed that the secondary component of BM Orionis is a disk of total mass one to three solar masses. There are difficulties in reconciling the light curve with the fact that only a B-type spectrum is visible if the system is supposed to consist of two normal spherical stars. Hall deduces a mass for the disk of from one to three solar masses, but it is not clear whether the disk is homogeneous or contains a 
normal central star surrounded by a not very massive, but optically thick disk.

As was apparent in Section 2, the dimensions of streams are very much less wellknown than those of disks, and the masses are correspondingly uncertain. In most systems, however, the volume of the stream seems to be much less than that of the disk, and since the densities are similar, the masses of the streams must be small compared with those of the disks. Little, if anything is known about the masses of clouds Although they probably contain relatively little mass at any one time, because of their low densities, they may be the means by which some of the mass of a binary system is completely lost into space. The available data indicate that the amount of mass between the components of a binary system is nearly always a very small fraction of the total mass of the system.

\section{Ionization and Temperature of Circumstellar Matter}

Circumstellar matter must be very far from local thermodymanic equilibrium, and therefore its temperature, in any given system, cannot be uniquely defined. In any system in which the matter shows an identifiable spectrum, however, some estimate of the degree of ionization, and hence of the ionization temperature, can be made, although this will necessarily be different from the temperature defined by the mean energy of individual atoms. For example, in $\beta$ Lyrae, different portions of the stream appear to have different temperatures. Thus the expanding shell (cloud) is given a spectral type of B2 or B5 compared with the central star's type of B8. The red-displaced satellite lines are described as resembling an A2 spectrum (Sahade et al., 1959), or more precisely, mainly on the basis of the ratio of the intensities of the lines of Fe I to those of Fe II, intermediate in type between $\alpha$ Cygni (A2) and $\alpha$ Persei (F5) (Struve and Zebergs, 1959), while the violet-displaced satellite lines correspond to a distinctly higher stage of ionization. Thus different ionization temperatures can be assigned to the various parts of the circumstellar matter in $\beta$ Lyrae, although the different sets of lines do show anomalous intensities. For example, the red-satellite lines include lines of helium that should scarcely be visible at all in an A2 spectrum, and the early type of the shell lines no doubt represents an effect of the reduced electron pressure, rather than of higher temperature. Thus, the example of $\beta$ Lyrae both illustrates what can be done in this way, and shows the difficulty of assigning a meaningful temperature to circumstellar matter.

Heiser (1961) has tried to estimate the excitation temperature of the stream in V 367 Cygni at different phases. At phases 0.18 and $0^{P} .46$ he finds an excitation temperature of $6700 \mathrm{~K}$, while at phase 0.78 it is only $5400 \mathrm{~K}$. It is quite conceivable that the stream should appear to have different temperatures at different phases, for one may be looking at different parts of the stream which may be at different distances from the exciting star (probably about A2 in V 367 Cygni), but it is not clear whether this reported difference is significant. In U Cephei, also, there is some evidence that a part of the stream has a 'temperature' similar to that of the G-type component, while most of it seems to display the same ionization as does the B-type component. 
Not much work has been done on the temperatures of streams in other Algol-type systems, although, as already pointed out in Section 2, the presence of a helium line in the spectrum of the stream in Algol itself suggests an ionization in the stream similar to that in the atmosphere of the primary component.

Hutchings and Wright (1971) derive values for the excitation temperature at different levels in the envelope surrounding the secondary star of VV Cephei. They believe this star to be of late $\mathrm{O}$ or early $\mathrm{B}$ spectral type. They find an excitation temperature that varies from $16000 \mathrm{~K}$ at a distance from the secondary star equal to 0.01 times the separation of the two stars, to $7000 \mathrm{~K}$ at a distance of 0.12 times the separation. These figures are not obtained directly from observation, but by fitting a model to the emission-line profiles.

Kraft (1959) attempted to determine temperatures for the assumed nova component of DQ Herculis and the surrounding disk, from their combined colour. He found that the best fit was obtained if he assumed the nova component (which contributed $25 \%$ of the visual light) has a temperature of $80000 \mathrm{~K}$ and an electron temperature of $40000 \mathrm{~K}$ for the disk. Other arguments (from the ionization equilibrium of helium and hydrogen) indicate even higher temperatures. Similar high temperatures were found for circumstellar matter in T Coronae Borealis (Kraft, 1958).

Warner and Nather (1971) have also estimated a temperature of $50000 \mathrm{~K}$ for the circumstellar matter in U Geminorum, but this is confined to the hot spot that they believe is formed by the collision of the stream and the disk, and which they estimate to be confined to an area of about $3 \times 10^{9} \mathrm{~cm}$ diameter. Their estimate of temperature, however, depends partly upon the very high densities they have assumed for the stream and disk, although not sensitively so since it is the ratio of the two densities that is important. Nevertheless, Smak (1971a) has attempted to estimate the temperatures of hot spots in several eruptive binaries from their colours (which can be determined from the colour change during eclipse if the eclipse is essentially of the hot spot) and finds the surprisingly low range $7000 \mathrm{~K}$ to $18000 \mathrm{~K}$. From this result, he deduces equivalent black-body temperatures for the disks of $12000 \mathrm{~K}$ or less. Thus there is much uncertainty in the estimated temperatures of both hot spots and disks in eruptive binaries.

Another attempt to estimate the ionization temperature of a disk in a long-period system has been made by Günther (1959) for SX Cassiopeiae. He has attempted to derive an ionization temperature by means of Saha's law, and finds $11500 \mathrm{~K}$ although the effective temperature of the central star is only $8400 \mathrm{~K}$. As he estimated a fairly high density for the disk (about $10^{12}$ electrons $\mathrm{cm}^{-3}$ ) this temperature seems to be anomalously high. This, however, probably again indicates the difficulty of defining the temperature of circumstellar matter in a meaningful way.

Related to questions of temperature and ionization is the occurrence of flaring in close binary systems. 'Flares' have been observed in W Ursae Majoris systems (Huruhata, 1952; Eggen, 1948) and in YY Geminorum (Bopp and Moffet, 1971) who now relate them to circumstellar matter in this latter system (private communication). Changes in the equivalent width of $\mathrm{H} \alpha$ in the spectrum of $\mathrm{U}$ Cephei also suggest some 
sort of flare activity in that system. The most important recent evidence, however, is undoubtedly the radio observations of Algol, cited in Section 1, that strongly suggest a flare-like process is taking place in that system.

Hjellming (1972) has made some estimates of the densities and temperatures needed to produce the observed radio fluxes. He finds that the emitting region must have a diameter in the range $0.1 \mathrm{AU}$ to $13 \mathrm{AU}$, a temperature between $2.5 \times 10^{5} \mathrm{~K}$ and $2 \times 10^{9} \mathrm{~K}$ and electron densities between $6 \times 10^{7} \mathrm{~cm}^{-3}$ and $9 \times 10^{10} \mathrm{~cm}^{-3}$. These figures suggest that the 'flares' do not occur in the stream or disk, but in the cloud.

There is a need to be careful about terminology here. The word 'flare' immediately suggests a solar flare, and there is a temptation to link the transfer of matter within these systems to solar-type activity on the mass-losing component. Judged as a means of mass transfer, however, a normal solar flare is an inefficient mechanism. It accelerates a very small amount of matter to a much higher velocity than is needed to effect mass transfer, and gives it a large amount of radiant energy as well. An ideal masstransfer mechanism would expend the same amount of energy on very much more matter. The activity on a stellar surface that leads to mass transfer is probably different in kind from solar activity, but it may well take place in surges that give rise to some of the phenomena associated with solar flares. It may be these phenomena that have been observed. The situation is complicated by the use of the same term to describe flare stars which may also be quite different phenomenon. It might be well to use the term 'flare' circumspectly, and perhaps in our discussions we can think of a better term.

\section{Solid Circumstellar Matter}

Some binary systems may contain solid circumstellar matter. Clouds of solid particles have been proposed to explain light variation of the type found in $\mathrm{R}$ Coronae Borealis, and there seems no fundamental reason why they should not exist in binary systems, especially in those containing only cool stars. Kopal $(1954,1971 \mathrm{~b})$ has suggested that a ring of solid particles surrounds the invisible secondary component of $\varepsilon$ Aurigae (although a disk of such particles was suggested even earlier - Lüdendorff, 1924; Schoenberg and Jung, 1938). Wilson (1971) developed this idea further, particularly with reference to Cameron's (1971) proposal that the system contains a black hole. This latter proposal is not necessary for an adequate explanation of $\varepsilon$ Aurigae (Demarque and Morris, 1971), and the scientific attitude toward it would seem to be to maintain a cautious. economy of hypotheses. Demarque and Morris do not even favour the hypothesis of solid particles, preferring instead a hot gaseous ring. If the secondary component really is a hot star, as they propose, then solid particles within the system would be unlikely although it has recently been suggested that they formed around Nova Serpentis 1970 (Kleinmann et al., private communication to J. B. Hutchings). The matter cannot yet be regarded as resolved. Evans (1971) has suggested that there are clouds of solid particles in the system of CC Eridani (and in other similar dwarf, late-type spectroscopic binaries) although an alternative explanation in terms of star spots has been proposed (Krzeminski, 1969). A disk of solid particles has also 
been proposed for another system containing late-type dwarfs, namely RT Lacertae (Hall and Taylor, 1971). Further investigation of many of these systems seems desirable.

\section{Velocities Within Circumstellar Matter}

Velocities are the only clue we have to the dynamics of circumstellar matter. Since they must be determined spectroscopically, we can know only the line-of-sight component of the velocity at any given orbital phase of a binary system. As the orbital phase changes, the observed change in radial velocity is produced not only by the changing aspect of the system, but also, often, by a change in the portion of the stream exposed to view.

The velocities of rotation of the disks can be completely observed. Struve (1950) frequently drew attention to the relation

$$
V^{3} \propto 1 / P
$$

between the rotational velocity of a disk, $V$, and the orbital period, $P$. Huang and Struve (1956) showed that a relation of the form

$$
V^{3}=(1 / P) f\left(m_{1}, m_{2}, a, \varrho\right)
$$

was to be expected from particle dynamics, where $m_{1}$ and $m_{2}$ are the masses of the two stars in the system, and $a$ and $\varrho$ have already been defined in Section 2 . The function $f$ is given by

$$
f\left(m_{1}, m_{2}, a, \varrho\right)=2 \pi G m_{1}\left(\frac{m_{1}}{m_{1}+m_{2}}\right)^{1 / 2}(a / \varrho)^{3}
$$

which is not expected to vary widely from system to system (i.e. $m_{1}, a \mid \varrho$, and $\left\{m_{1} \mid\right.$ $\left.\left(m_{1}+m_{2}\right)\right\}^{1 / 2}$ are supposed to be similar in all systems) so the observed relation appeared to have some theoretical basis. Most of the observational data available to Struve concerned Algol-type systems, in which, as far as we know, these three quantities can be assumed to be similar. We now have data for eruptive binaries, and very large systems like $\varepsilon$ Aurigae and VV Cephei, and, surprisingly, they all fit a similar, but different, relation, namely

$$
V^{4.6} \propto 1 / P
$$

(see Figure 4). The rings in eruptive binaries rotate more slowly than Struve's formula predicted. In Section 2, it was shown that $\varrho / a$ is fairly constant from system to system, over this wide range of systems that has now been observed, but of course there is a wide variation of $m_{1}$. It is surprising that the results from such diverse systems should fit any relation, and this suggests that the relation actually found has some significance. Smak's (1969) result that the observed rotational velocities of circumstellar disks are underestimates of the true velocities will not affect the form of the relation between $V$ and $P$. This observed relation may be a definite indication of the failure of particle dynamics within disks, since it cannot be derived under the assumptions of particle 


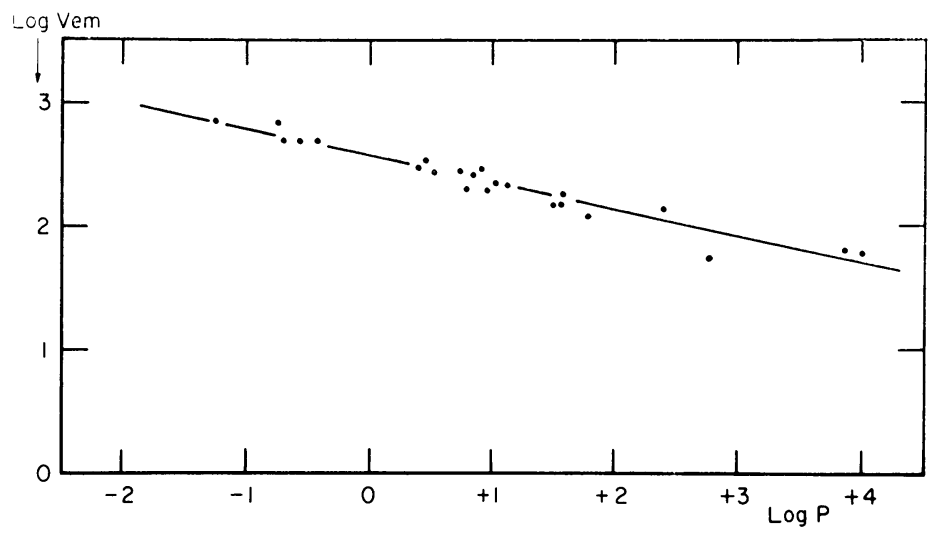

Fig. 4. Empirical relation between the velocity of rotation of the disk $\left(V_{\mathrm{em}}\right)$ and the orbital period

dynamics. The failure of these assumptions within disks is hardly surprising, especially since some disks are observed in systems (U Cephei, U Sagittae, RW Tauri) in which, according to those assumptions, disks should not form at all (Kruzewski, 1967).

The existence of this apparent relation between $V$ and $P$ in systems of remarkably different characteristics is one aspect of a surprising uniformity within these systems. Circumstellar matter is found in systems of vastly different scale, from the eruptive binaries with orbital periods of a few hours, to supergiant systems like VV Cephei and $\varepsilon$ Aurigae with their periods of $20 \mathrm{y}$ and $27 \mathrm{y}$ respectively. The linear dimensions of the systems vary correspondingly. The short-period systems contain white dwarfs, or very hot subdwarfs, the Algol-type systems contain main-sequence B-type stars, while other systems contain enormously large, late-type supergiants. There seems to be no a priori reason to suppose that circumstellar matter will behave in the same way in all these diverse systems, and yet the models proposed to explain the phenomena have many points of similarity, of which the relation between $V$ and $P$ is only the most striking. A possible exception to this rule is the systems containing very massive, early-type stars, in which the motion of circumstellar matter may be dominated by mass loss from one or both components (Sahade, 1962).

The velocities of streams are harder to deduce, but at some phases (particularly just before primary eclipse, at least in the Algol-type systems) the stream can be observed in projection on the primary star, with its velocity virtually entirely in the radial direction. Typical values for the velocity then seem to be several hundred $\mathrm{km} \mathrm{s}^{-1}$ (about $450 \mathrm{~km} \mathrm{~s}^{-1}$ in $\mathrm{U}$ Cephei). These values coincide with the expected terminal velocity of matter falling on to the primary star. This terminal velocity is fairly insensitive to the assumed velocity of ejection from the secondary star, and we cannot deduce very much about the ejection velocity, unless the whole trajectory can be determined. It is for this reason that the question of the value of particle-dynamics trajectories is hard to settle observationally. There is no evidence from the streams (as distinct from the disks) to show that such trajectories are inadequate. 
Most of the material on velocities of clouds has been summarized in the earlier sections. Some clouds seem to be expanding, and some do not. Others seem to be rotating. Further observations are desirable to try to establish the rate of mass loss from systems containing clouds.

\section{Stability of Circumstellar Features}

An almost untouched question is that of the stability of streams, disks, and clouds. If few systems have been examined in sufficient detail for a proper determination of the circumstellar properties at one epoch, none at all, as far as I know, has been observed in necessary detail at more than one epoch.

Three kinds of changes may be expected in circumstellar features:

(i) apparent changes with changing aspect of the system,

(ii) short-term changes (probably fluctuations about a mean) within a few orbital periods, or less,

(iii) long-term changes within the system, possibly of evolutionary interest.

Changes of type (i) are only apparent, and should be periodic. In practice, however, they may be difficult to separate from some changes of type (ii). Changes of type (iii) would be difficult to observe, requiring observations from at least three epochs. Nevertheless, Sahade has suggested that changes in the streams of Plaskett's star may be of this type. Larsson-Leander (1969) has reported that $\beta$ Lyrae appeared to become slightly fainter and slightly redder over an interval of ten years - a change that would fit in with our evolutionary ideas, if $\beta$ Lyrae is indeed in the rapid phase of mass exchange. Guinan (private communication) believes changes have occurred in the emission in the spectrum of $\beta$ Lyrae even within the past year. Walter (1970) has reported real changes in the light curve of RV Ophiuchi, over an interval of $50 \mathrm{yrs}$. These could be of type (iii), but Walter himself seems to prefer to regard them as changes of type (ii). Small changes in the size and location of a stream could, he believes, have a considerable effect on the light curve.

This section, however, is primarily concerned with changes of type (ii), so far as they can be distinguished from those of type (i). The reality of changes of type (iii) must for a long time remain speculative. The emission observed in RW Tauri at primary minimum is variable in intensity, and sometimes absent (Joy, 1942, 1947). Similar variations are found in U Cephei and U Sagittae, in each of which the emission has been looked for several times, and found only once. This is so even though observations have been carefully timed to achieve the maximum probability of detection of any ring. An obvious explanation is that the disk producing the emission is sometimes absent. This is backed up by theoretical investigations that make the formation of a disk seem rather unlikely in these particular systems (Kruzewski, 1967) and also suggest that disks will speedily collapse if the streams that feed them are cut off (Gorbatskij, 1969). Gorbatskij's calculations referred to U Geminorum systems, and whether they can also be applied to Algol-type systems I do not know. They do, however, point to a problem in the interpretation of $U$ Cephei, and that is the contrast 
between the apparent permanence of photometric effects, and the transience of spectroscopic effects, of circumstellar matter. If the disturbances of light and velocity curves are correctly interpretated as the result of circumstellar matter, we have here a paradox since the light curve shows that the stream has been fundamentally constant for at least fifty years, while the velocity curve shows variations from epoch to epoch and a disk has been seen only once in the system. Spectroscopic observations, of course, are more senstive to changes in the stream than broad-band photometric observations are, but the complete disappearance of the disk is hard to explain if the stream has indeed been virtually constant. One possible explanation is that changes occur in the ionization of the stream and disk that would greatly affect the observable emission, but hardly effect the efficiency of the circumstellar matter in electron scattering - which is what determines its effect on the light curve. The observed emission lines could be produced by about one atom in every $10^{4}$. If the normal state of the matter were complete ionization, the disk would be invisible in emission. If occasionally a relatively few ions recombined with electrons, then emission lines would become briefly visible. A difficulty is to imagine a mechanism that would achieve this. Flaring activity would be expected to operate the other way.

Other systems in which the disk is permanently present, usually of long period, (e.g. SX Cassiopeiae) may well exhibit changes of extent and structure of the disks, but insufficient observations have yet been made to establish any. One of my present aims is to build up a bank of observations of such systems that may be useful in the future elucidation of this problem.

Some possible changes in eruptive binaries have been mentioned by Kraft (1958, 1959) but the general situation remains that not enough observations have yet been made. Heiser (1961) also discusses changes in V 367 Cygni, that may be real changes of type (ii).

\section{Conclusions}

The aim of this review has been to investigate what is already known about the physical properties of circumstellar matter in order to find out what constraints are placed on any theory by the observational results at present aviable. This has been shown to be a difficult task, because quantitative observational estimates are few, and not always accordant. I have deliberately avoided any detailed discussion of the dynamics of circumstellar matter, believing this to be properly a part of the area that Dr. Huang is to review. The following conclusions summarize the result of our inquiry.

(a) The density of circumstellar matter is probably about the best determined quantity at present. Several arguments lead to densities for the streams of about $10^{13}$ particles $\mathrm{cm}^{-3}$. There is no doubt a real variation between systems, as well as observational uncertainty in this figure. Estimates as high as $10^{16}$ or $10^{17}$ particles $\mathrm{cm}^{-3}$ have been suggested for $U$ Geminorum. While it cannot be ruled out that the circumstellar matter in this system is exceptional, these figures are so much higher than the average, even in similar systems, that their derivation should be scrutinized very carefully before 
they are accepted. It seems likely that their derivation is based on a misinterpretation of the observed period change. Disks around one component of the binary probably have densities similar to those of the streams in the same systems. Clouds are less dense, being more remote from the stellar components in the binary system. Their densities appear to be $10^{11}$ particles $\mathrm{cm}^{-3}$, or less.

(b) Velocities of the matter in streams can usually be observed only in the neighbourhood of the primary star, where they appear to be several hundred $\mathrm{km} \mathrm{s}^{-1}$. Such values are consistent with what would be expected if mass is being transferred from one star to the other, largely or solely under the control of gravitation (that is, hydrodynamic and magnetic effects appear not to be detectable above the limits set by observational uncertainty). The very existence of disks, however, requires a modification of particle dynamics, at least in some systems. The observed velocities within the disks (also several hundred $\mathrm{km} \mathrm{s}^{-1}$ - except in the very long-period systems) may not be explicable simply in terms of particles describing orbits around the primary star.

(c) Dimensions of streams are very hard to estimate. The disks appear to have radii of several times the radius of their central star, or (in Algol-type systems) about half the separation between the stars. The masses of disks are very small, about $10^{-9} \mathrm{M}_{\odot}$. Sometimes they may be larger, but seldom if ever massive enough for period changes to be explicable as an interaction between rotating disk and central star, as proposed by Smak (1971b). This kind of interaction is ruled out by the observations, even if the high circumstellar densities proposed by Warner and Nather (1971) for U Geminorum are accepted.

(d) Temperatures of circumstellar features are hard either to define or determine. Some estimate can often be made of the approximate degree of ionization. These estimates show that streams have temperatures less than or equal to that of the hotter star in the system. Although this conclusion is to be expected, it is perhaps of some value to show that it has observational support. Where two streams, or a stream and a disk collide, a hot spot may be formed. Some estimates of the temperatures of these spots make them very hot - about $50000^{\circ} \mathrm{K}$ - but there is considerable uncertainty just how hot they are. There is both optical and radio evidence for flare-like activity in systems containing circumstellar matter. It is not yet clear whether the seat of the activity is the matter itself, or one of the component stars. In either case, it could be associated with temporary changes in the degree of ionization of the circumstellar matter. Finally, under this head, the possibility (and significance) of solid circumstellar matter perhaps deserves more theoretical attention.

(e) The stability of many circumstellar features is unknown. The available observational evidence is inadequate and confusing.

This is the best that the observers have done so far. Many of the observations required are difficult to make and present a real challenge. The interesting binary systems are very faint, at least at the phases that are of most importance for the study of circumstellar matter. Good high-dispersion spectrograms of many of these systems have been almost impossible to obtain, until recently, and even now the radio observations of binary systems are being made at the limit of the detecting apparatus. Broad- 
band photometry, while useful, is not sensitive to the effects of circumstellar material. New observational techniques providing high time-resolution, and observation from above the Earth's atmosphere will certainly improve our knowledge, as the first X-ray and far UV observations have already shown. They may even revolutionize our concepts. Nevertheless the conventional techniques have not yet been utilized to their full, and are capable of giving us much more information about many systems. This field of circumstellar matter is challenging to observers of all kinds, and there is much work to be done.

\section{Acknowledgments}

I am grateful to my colleagues Drs. R. W. Hiltditch, J. B. Hutchings, and G. J. Odgers for critical comments on an earlier draft of this paper. Figures 2 and 4 are reproduced by kind permission of Pergamon Press.

\section{References}

Andrews, P. J.: 1967, Astrophys. J. 147, 1183.

Baldwin, B. W.: 1972, M.Sc. Thesis, University of Victoria (unpublished).

Batten, A. H.: 1970, Publ. Astron. Soc. Pacific 82, 574.

Batten, A. H.: 1973, Binary and Multiple Systems of Stars, Pergamon Press, Oxford, pp. 166-221.

Bierman, P.: 1971, Astron. Astrophys. 10, 205.

Bolton, C. T.: 1972a, Nature 235, 271.

Bolton, C. T.: 1972b, J. Roy. Astron. Soc. Can. 66, 219.

Bopp, B. W. and Moffet, T. J.: 1971, Astrophys. J. Letters 168, L117.

Braes, L. L. E. and Miley, G. K.: 1972, Nature 235, 273.

Brown, R. H., Davis, J., and Herbison-Evans, D.: 1970, Monthly. Notices Roy. Astron. Soc. $148,103$.

Burbidge, E. M., Lynds, C. R., and Stockton, A. N.: 1967, Astrophys. J. Letters 150, L95.

Cameron, A. G. W.: 1971, Nature 229, 178.

Coyne, G. V.: 1970, Ric. Astron. Specola Astron. Vatic. 8, 85.

Crawford, J. A. and Kraft, R. P.: 1956, Astrophys. J. 123, 44.

Demarque, P. and Morris, S. C.: 1971, Nature 230, 516.

Eggen, O. J.: 1948, Astrophys. J. 108, 15.

Evans, D. S.: 1971, Monthly Notices Roy. Astron. Soc. 154, 329.

Fletcher, E. S.: 1964, Astron. J. 69, 357.

Gorbatskij, V. G.: 1969, Astrophys. Space Sci. 3, 179.

Günther, O.: 1959, Astron. Nachr. 285, 97 and 105.

Hall, D. S.: 1969, Bull. Am. Astron. Soc. 1, 345.

Hall, D. S.: 1971, Veröffentl. Remeis-Sternw. Bamberg 9, 217.

Hall, D. S. and Garrison, L. M.: 1972, Publ. Astron. Soc. Pacific 84, 552.

Hall, D. S. and Taylor, M. C.: 1971, Bull. Am. Astron. Soc. 3, 12.

Hansen, H. K. and McNamara, D. H.: 1959, Astrophys. J. 130, 791.

Heiser, A. M.: 1961, Astrophys. J. 134, 568.

Herbig, G. H., Preston, G. W., Smak, J., and Paczynski, B.: 1965, Astrophys. J. 139, 306.

Hjellming, C.: 1972, Nature Phys. Sci. 238, 52.

Hughes, V. A. and Woodsworth, A.: 1972, Nature Phys. Sci. 236, 42.

Huang, S.-S. and Struve, O.: 1956, Astron. J. 61, 300.

Huruhata, M.: 1952, Publ. Astron. Soc. Pacific 64, 200.

Hutchings, J. B.: 1970, Monthly Notices Roy. Astron. Soc. 147, 367.

Hutchings, J. B. and Wright, K. O.: 1971, Monthly Notices Roy. Astron. Soc. 155, 203.

Joy, A: H.: 1942, Publ. Astron. Soc. Pacific 54, 35.

Joy, A. H.: 1947, Publ. Astron. Soc. Pacific 59, 171.

Koch, R. H.: 1972, Astron. J. 77, 500. 
Kondo, Y.: 1971, Veröffentl. Remeis-Sternw. Bamberg 9, 298.

Kondo, Y., Giuli, R. T., Modisette, J. L., and Rydgren, A. E.: 1972, Astrophys. J. $176,153$.

Kopal, Z.: 1954, Observatory 74, 14.

Kopal, Z.: 1971a, Publ. Astron. Soc. Pacific 83, 521.

Kopal, Z.: 1971b, Astrophys. Space Sci. 10, 332.

Korsch, D. and Walter, K.: 1969, Astron. Nachr. 291, 231.

Kraft, R. P.: 1958, Astrophys. J. 127, 625.

Kraft, R. P.: 1959, Astrophys. J. 130, 110.

Kraft, R. P.: 1972, Bull. Am. Astron. Soc. 4, 219.

Kraft, R. P. and Demoulin, M.-H.: 1967, Astrophys. J. Letters 150, L183.

Kristian, J;, Sandage, A. R., and Westphal, J. A.: 1967, Astrophys. J. Letters 150, L99.

Kruszewski, A.: 1967, Acta Astron. 17, 297.

Krzeminski, W.: 1969, in S. S. Kumar (ed.), Low Luminosity Stars, Gordon and Breach, New York, p. 57.

Krzeminski, W. and Kraft, R. P.: 1964, Astrophys. J. 140, 921.

Kuiper, G. P.: 1941, Astrophys. J. 93, 133.

Larsson-Leander, G.: 1969, Arkiv Astron. 5, 253.

Lüdendorff, H.: 1924, Sitzber. Deut. Akad. Wiss. Berlin 49.

Morton, D. C.: 1967a, Astrophys. J. 147, 1017.

Morton, D. C.: 1967b, Astrophys. J. 150, 535.

Morton, D. C., Jenkins, E. B., and Bohlin, R. C.: 1968, Astrophys. J. 154, 661.

Murdin, P. and Webster, B. L.: 1971, Nature 233, 110.

Murdin, P. and Webster, B. L.: 1972, Nature 235, 37.

Plavec, M.: 1968, Bull. Astron. Inst. Czech. 19, 11.

Plavec, M., Sehnal, L., and Mikuláš, J.: 1964, Bull. Astron. Inst. Czech. 15, 171.

Plavec, M. and Kříž, S.: 1965, Bull. Astron. Inst. Czech. 16, 297.

Plavec, M. and Harmanec, P.: 1972, Comm. 27 IAU, Inf. Bull. Var. Stars, No. 163.

Prendergast, K. H.: 1960, Astrophys. J. 132, 162.

Prendergast, K. H. and Burbidge, G. R.: 1968, Astrophys. J. Letters 151, L83.

Sahade, J.: 1962, in J. Sahade (ed.), Symposium on Stellar Evolution, La Plata, p. 185.

Sahade, J., Huang, S.-S., Struve, O., and Zebergs, V.: 1959, Trans. Am. Phil. Soc. New Series 49 , Part I.

Schoenberg, E. and Jung, B. : 1938, Astron. Nachr. 265, 221.

Shulov, O. S.: 1967, Astrofizika 3, 233.

Skilling, W. T. and Richardson, R. S.: 1947, Astronomy, Henry Holt and Co., New York.

Smak, J.: 1969, Acta Astron. 19, 155.

Smak, J.: 1971a, Veröffentl. Remeis-Sternw. Bamberg 9, 248.

Smak, J.: 1971b, Acta Astron. 22, 1.

Sobouti, Y.: 1970, Astron. Astrophys. 5, 149.

Struve, O.: 1950, Stellar Evolution, Princeton Univ. Press, Princeton.

Struve, O.: 1960, Sky Telesc. 19, 276.

Struve, O. and Sahade, J.: 1957, Publ. Astron. Soc. Pacific 69, 41.

Struve, O., Sahade, J., and Huang, S.-S.: 1957, Publ. Astron. Soc. Pacific 69, 342.

Struve, O. and Zebergs, V.: 1959, Astrophys. J. 130, 817.

Thackeray, A. D.: 1959, Monthly Notices Roy. Astron. Soc. 119, 629.

Thackeray, A. D.: 1971, Monthly Notices Roy. Astron. Soc. 154, 103.

Van den Bergh, S.: 1972, Nature 235, 271.

Wade, C. M. and Hjellming, C.: 1972, Nature 235, 270.

Walker, M. F. and Herbig, G. H.: 1954, Astrophys. J. 120, 278.

Walter, K.: 1970, Astron. Astrophys. 5; 140.

Walter, K.: 1971a, in Analytical Procedures for Eclipsing Binary Light Curves, IAU Colloquium No. 16 (Philadelphia); in press.

Walter, K.: 1971b, Astron. Astrophys. 13, 249.

Walter, K.: 1972, in press.

Warner, B. and Nather, R. E.: 1971, Monthly Notices Roy. Astron. Soc. 152, 219.

Wilson, R. E.: 1971, Astrophys. J. 170, 529.

Woolf, N. J.: 1965, Astrophys. J. 141, 155. 